\title{
Implementing Change in the Heat of the Moment
}

\author{
Chad H Hochberg, MD, Michelle N Eakin, PHD, David N Hager, MD, PHD*
}

Division of Pulmonary \& Critical Care Medicine, Johns Hopkins University, Baltimore, Maryland.

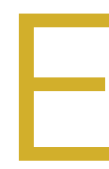

arly in the severe acute respiratory syndrome coronavirus 2 (SARS-CoV-2) pandemic, the World Health Organization issued guidance for coronavirus disease 2019 (COVID-19) management. ${ }^{1}$ Based on a high intubation rate among 12 subjects with Middle Eastern respiratory syndrome, noninvasive ventilation (NIV) was discouraged. ${ }^{2}$ While high-flow nasal oxygen (HFNO) was recognized as a reasonable strategy to avoid endotracheal intubation, ${ }^{1}$ uncertainty regarding the potential of both therapies to aerosolize SARS-CoV-2 and reports of rapid, unexpected respiratory decompensations were deterrents to use. ${ }^{3}$ As hospitals prepared for a surge of patients, reports of SARS-CoV-2 transmission to healthcare personnel also emerged. Together, these issues led many institutions to recommend lower than usual thresholds for intubation. This well-intentioned guidance was based on limited historical data, a rapidly evolving literature that frequently appeared on preprint servers before peer review, or as anecdotes on social media.

As COVID-19 caseloads increased, clinicians were immediately faced with patients who rapidly reached the planned intubation threshold, but also looked very comfortable with minimal to no use of accessory muscles of respiration. In addition, the pace of respiratory decompensation among those who ultimately required intubation was slower than expected. Moreover, intensive care unit (ICU) capacity was stretched thin, raising concern for an imminent need for ventilator rationing. Lastly, the risk of SARS-CoV-2 transmission to healthcare workers appeared well-controlled with the use of personal protective equipment. ${ }^{4}$

In light of this accumulating experience, sites worldwide evolved quickly from their initial management strategies for COVID-19 respiratory failure. However, the deliberate process described by Soares et al in this issue of the Journal of Hospital Medicine is notable. ${ }^{5}$ Their transition towards the beginning of the pandemic from a conservative early intubation approach to a new strategy that encouraged use of NIV, HFNO, and self-proning is described. They were motivated by reports of good outcomes using these interventions, high mortality in intubated patients, and reassurance that aerosolization of respiratory secretions during NIV and HFNO was comparable to regular nasal cannula or face mask oxygen. ${ }^{3}$ The new protocol was defined and rapidly deployed over 4 days using multipronged communication from project and institutional leaders via in person and electronic means (email, Whatsapp, GoogleDrive). To

*Corresponding Author: David N Hager, MD, PHD; Telephone: 410-614-6292; Email: dhager1@jhmi.edu; Twitter: @davidnhager.

Received: November 3, 2020; Revised: November 4, 2020;

Accepted: November 5, 2020

๑ 2020 Society of Hospital Medicine DOI 10.12788/jhm.3565 facilitate implementation, COVID-19 patients requiring respiratory support were placed in dedicated units with bedside flowsheets for guidance. An immediate impact was demonstrated over the next 2 weeks by a significant decrease in use of mechanical ventilation in COVID-19 patients from $25.2 \%$ to $10.7 \%$. In-hospital mortality, the primary outcome, did not change, ICU admissions decreased, as did hospital length of stay (10 vs 8.4 days, though not statistically significant), all providing supportive evidence for relative safety of the new protocol.

Soares et al exemplify a nimble system that recognized planned strategies to be problematic, and then achieved rapid implementation of a new protocol across a four-hospital system. Changes in medical practice are typically much slower, with some studies suggesting this process may take a decade or more. Implementation science focuses on translating research evidence into clinical practice using strategies tailored to particular contexts. The current study harnessed important implementation principles to quickly translate evidence into practice using effective engagement and education of key stakeholders across specialties (eg, emergency medicine, hospitalists, critical care, and respiratory therapy), the identification of pathways that mitigated barriers, frequent re-evaluation of a rapidly evolving literature, and an open-mindedness to the value of change. ${ }^{6}$ As the pandemic continues, traditional research and implementation science are critical not only to define optimal treatments and management strategies, but also to learn how best to implement successful interventions in an accelerated manner. ${ }^{7}$

Disclosures: The authors reported no conflicts of interest.

Funding: Dr Hochberg is supported by a National Institutes of Health training grant (T32HL007534).

\section{References}

1. World Health Organization. Clinical management of severe acute respiratory infection when novel coronavirus (1019-nCoV) infection is suspected: interim guidance, 28 January 2020. Accessed October 25, 2020. https://apps.who. int/iris/handle/10665/330893

2. Arabi YM, Arifi AA, Balkhy HH, et al. Clinical course and outcomes of critically ill patients with Middle East respiratory syndrome coronavirus infection. Ann Intern Med. 2014;160:389-397. https://doi.org/ 10.7326/M13-2486

3. Westafer LM, Elia T, Medarametla V, Lagu T. A transdisciplinary COVID-19 early respiratory intervention protocol: an implementation story. J Hosp Med. 2020;15:372-374. https://doi.org/10.12788/jhm.3456

4. Self WH, Tenforde MW, Stubblefield WB, et al. Seroprevalence of SARS CoV-2 among frontline health care personnel in a multistate hospital network - 13 academic medical centers, April-June 2020. MMWR Morb Mortal Wkly Rep. 2020;69:1221-1226. https://doi.org/10.15585/mmwr.mm6935e2

5. Soares WE III, Schoenfeld EM, Visintainer P, et al. Safety assessment of a noninvasive respiratory protocol. J Hosp Med. 2020;15:734-738. https://doi. org/ 10.12788/jhm.3548

6. Pronovost PJ, Berenholtz SM, Needham DM. Translating evidence into practice: a model for large scale knowledge translation. BMJ. 2008;337:a1714. https://doi.org/10.1136/bmj.a1714

7. Taylor SP, Kowalkowski MA, Beidas RS. Where is the implementation science? An opportunity to apply principles during the COVID19 pandemic. Online ahead of print. Clin Infect Dis. 2020. https://doi.org/10.1093/cid/ciaa622 